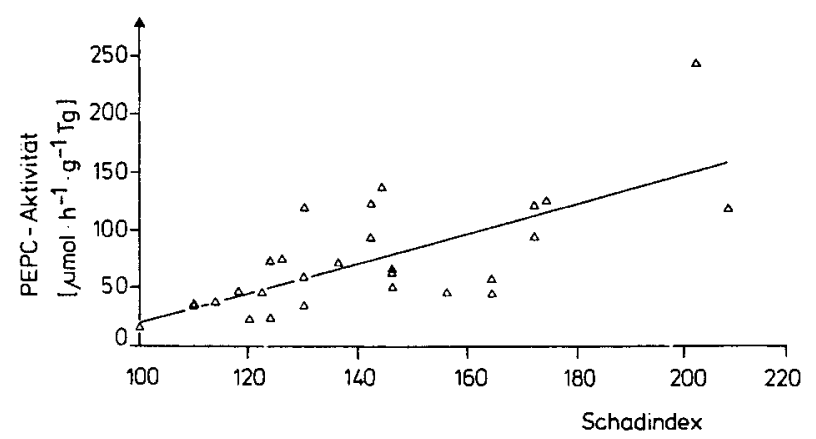

Abb. 2: Korrelation der PEPC-Aktivität und der differenzierten Schadklassifizierung (Schadindex) der Nadeln von 28 Fichten eines Standortes

Korrelationskoeffizient $\mathbf{r}=0.7$

\section{Die Funktion der erhöhten PEPC-Aktivität in den Nadeln geschädigter Fichten}

In der anaplerotischen $\mathrm{CO}_{2}$-Fixierung und der Bereitstellung von Oxalacetat als zentralem Stoffwechselmetaboliten ist eine der Hauptfunktionen der PEPC-Reaktion in Fichtennadeln zu sehen.

In Nadeln geschädigter Fichten ist der interne Stickstoffmetabolismus erhöht. Ebenso ist die Dunkelatmung bei geschädigten Fichten gesteigert. Dies bedingt einen erhöhten Bedarf an Kohlenstoffverbindungen des Citratcyclus. Normalerweise werden diese durch photsynthetische Prozesse zur Verfügung gestellt. Bei geschädigten Fichten sind jedoch sowohl die Komponenten der Thylakoidmembran als auch die Photosyntheserate gegenüber ungeschädigten Fichten erniedrigt. Die erhöhte PEPC-Aktivität in den Nadeln geschädigter Fichten könnte somit einen Reparaturmechanismus darstellen und den erhöhten Bedarf an Kohlenstoffverbindungen kompensieren.

\section{Danksagung}

Diese Studie wurde vom Bundesumweltamt Berlin, Förderungsnr. 10803 046/16, dem KfK-PEF Karlsruhe „Europäisches Forschungszentrum für $\mathrm{Maßnahmen} \mathrm{zur} \mathrm{Luft-}$ reinhaltung" Förderungsnr. 88/007/1A sowie dem Forschungsschwerpunkt des Landes Nordrhein-Westfalen „Luftverunreinigungen und Waldschäden" unterstützt.

\section{Literatur}

Diese Referenzen enthalten weitere Informationen und zablreiche Literaturangaben.

TIETZ, S.; A. WILD: Investigations on the phosphoenolpyruvate carboxylase activity of spruce needles relative to the occurrence of novel forest decline. J. Plant Physiol., 1990 (in press)

WILD, A.; W. FORSCHNER: Vergleichende physiologische und biochemische Untersuchungen an immissionsgeschädigten Fichten im $\mathrm{Zu}$ sammenhang mit den neuartigen Waldschäden an einem Standort im Nordschwarzwald. 6. Statuskolloquium des PEF vom 6. bis 8. März 1990. KFK-PEF 61, 297-309 (1990)

WILD, A.; W. ForsCHNER; V. SCHMITT: Physiologische, biochemische und cytomorphologische Untersuchungen an immissionsbelasteten Fichten. Forschungsendbericht für das Umweltbundesamt Berlin (1990)
Glossar. $\mathrm{C}_{3}$-Pflanzen bilden als Primärprodukt der $\mathrm{CO}_{2}-\mathrm{Fi}$ xierung 3-Phosphoglycerat. $-\mathrm{C}_{4}$-Pflanzen bilden als Primärprodukt der $\mathrm{CO}_{2}$-Fixierung die $\mathrm{C}_{4}$-Säuren Malat oder Aspartat. CAM-Pflanzen nehmen nachts $\mathrm{CO}_{2}$ von der Umgebung auf und $\mathrm{f}_{\mathrm{i}}$ xieren es auf den Stufen von $\mathrm{C}_{4}$-Säuren (Malat) vor. Im Licht (bei
Tag) wird das vor-fixierte $\mathrm{CO}_{2}$ in der Zelle frei und direkt in den Calvin-Zyklus eingeschleust. - Thylakoide sind die Photosynthesenmembranen in den Chloroplasten, in denen die Umwandlung der absorbierten Lichtenergie in chemische Energie stattfinder.

\title{
Immissionsökologisches Wirkungskataster Baden-Württemberg 1989
}

\section{- 6. Jahresbericht der Landesanstalt für Umweltschutz*}

\section{Einleitung}

Die Landesanstalt für Umweltschutz arbeitet seit 1984 im Rahmen des Immissionsökologischen Wirkungskatasters an einer landesweiten ökologischen Zustandserfassung Baden-Württembergs. Dieses natürliche Frühwarnsystem für die Gefährdung der Ökosysteme durch Schadstoffe aus der Luft basiert auf der Untersuchung sog. "Bioindikatoren“, Organismen, die auf Immissionen in typischer Art und Weise reagieren und somit eine Bewertung von Umweltrisiken für die Natur erlauben. Darüber hinaus ermöglicht das Wirkungskataster langfristig eine Qualitätskontrolle hinsichtlich der Auswirkungen von Emissionsminderungsmaßnahmen.

\section{Jahresbericht 1989}

Die Eröffnungsbilanz der ökologischen Zustandserfassung wurde mit dem Untersuchungsjahr 1988 abgeschlossen (LFU 1989; ZIM-
MERMANN 1989). Damit war der erste Teil eines Wirkungskatasters, die Ermittlung der Basiskenngröße, erfüllt. Das Jahr 1989 wurde zur Bearbeitung einer Reihe von Spezialfragen genutzt. Ziel war es, die vorhandenen Daten kritisch zu sichten, durch gezielte Forschungsprogramme zu vervollständigen und aus den vorhandenen Erkenntnissen Rückschlüsse für das weitere Untersuchungskonzept zu ziehen. Daneben erfolgte die Auswertung der noch nicht veröffentlichten Meßdaten aus den Jahren 1987/88 der Wald-Dauerbeobachtungsflächen mit der ersten Wiederholungsbeprobung zur Stoffakkumulation in der Kraut- und Baumschicht sowie den Ergebnissen des Rehnierenprojekts (Einzelheiten zu diesem Projekt sowie auch zum Gewässeruntersuchungsprogramm 1989 wollen bitte dem ausführlichen Bericht entnommen werden).

Die ersten Untersuchungsergebnisse zum Wirkungskataster ergaben bei den anorganischen Schadstoffkomponenten einen Belastungszustand der naturnahen Waldökosysteme auf allgemein niedrigem Niveau. Bestätigt werden diese Erkenntnisse durch die im vorliegenden Bericht zusammengestellten Daten der ersten Wie-

* Bezug durch: Landesanstalt für Umweltschutz Baden-Württemberg, Griesbachstr. 3, W-7500 Karlsruhe 21 
derholungsbeprobung der Kraut- und Baumschicht. Führten die Krautschichtmeßdaten zu keinen entscheidend neuen Aussagen zur Ökosystemgefährdung, zeigte die Stoffakkumulation der Baumschicht trotz des geringen Belastungsniveaus einen neuartigen Trend: die Standorte mit größeren Risiken für die Stabilität des Ökosystems liegen nicht mehr allein im nordwestlichen Teil des Landes. Ein neuer Schwerpunkt bildete sich in der östlich an den Großraum Stuttgart angrenzenden Region ( $\rightarrow A b b .1$ ).

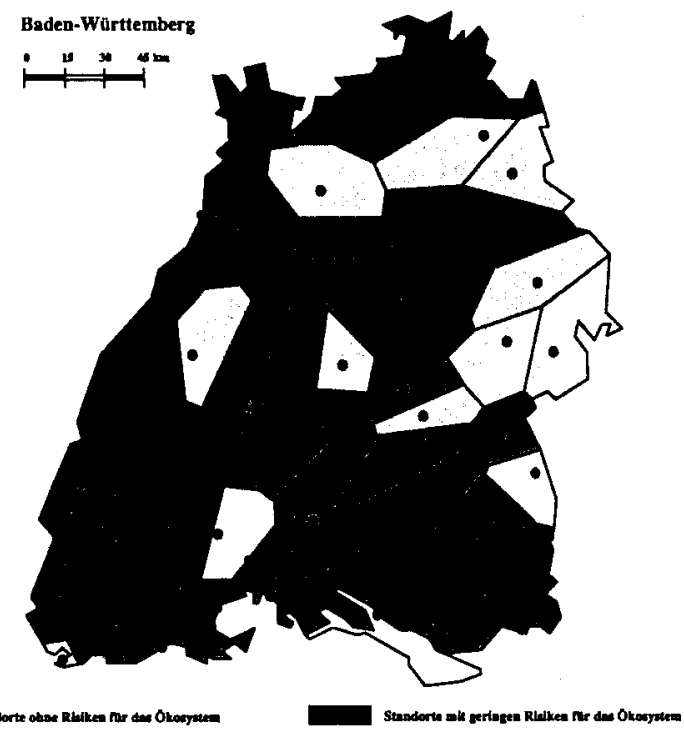

Abb. 1: Stoffakkumulation in der Baumschicht (1987/88)

Einschätzung der Ökosystemgefährdung aufgrund auffälliger Schwermetall- und/oder Nährstoffgehalte von Blattorganen

Anorganische Schadstoffkomponenten bilden nur einen relativ geringen Anteil der auftretenden Immissionen. Dies führte zu den Überlegungen, das Biomonitoring auf die Erfassung und Bewertung von organischen Luftschadstoffen auszuweiten (DEBUS et al., 1989). Erste Pilotprojekte zur Bioindikation von PAH und PCB mittels pflanzlicher Organismen wurden 1989 bereits erfolgreich durchgeführt. Geplant ist eine routinemäßige Erfassung der Konzentrationen dieser organischen Verbindungen an den landesweit eingerichteten Wald-Dauerbeobachtungsflächen für das Jahr 1991. - Die 1989 durchgeführten Spezialuntersuchungen beinhalteten u.a. für 22 ausgewählte Standorte eine Vegetationsaufnahme der epiphytischen Moose mit einer Überprüfung ihrer Eignung als Umweltindikatoren.

Vom Klon-Fichten-Meßnetz Baden-Württemberg liegen mittlerweile Ergebnisse aus drei aufeinanderfolgenden Jahren vor. Zunehmend hebt sich der nördliche Teil des Landes als belastetere Zone gegenüber der südlichen Region ab. Bei allen Untersuchungsperioden tritt der Großraum Mannheim/Karlsruhe hervor. Darüber hinaus zeigte der Raum östlich von Stuttgart eine steigende Belastungstendenz. Die Ergebnisse aus dem Jahr 1989 weisen einen deutlichen Zusammenhang zwischen der Lage der KlonFichten-Stationen der Kategorie „stärkere Belastung“ und dem Autobahnnetz von Baden-Württemberg auf ( $\rightarrow A b b$. 2).

Die seit 1984 durchgeführten Expositionen der Indikatorpflanzen zur Photooxidantienbelastung haben über die Jahre hinweg eine gleichbleibende Belastungsstruktur des Landes erkennen lassen.

Als regionale Wirkungsmeßprogramme wurden 1989 der Hochrhein sowie das Stadtgebiet Freiburg und Umland untersucht. Das Hochrheinprojekt ist ein kombiniertes Immissionsraster-, Wirkungs- und Bodenmeßprogramm. Für Freiburg wurden erstmalig umfangreiche Wirkungsuntersuchungen mit aktiven und passiven Verfahren durchgeführt. Neben Graskultur- und Pilzexponaten,

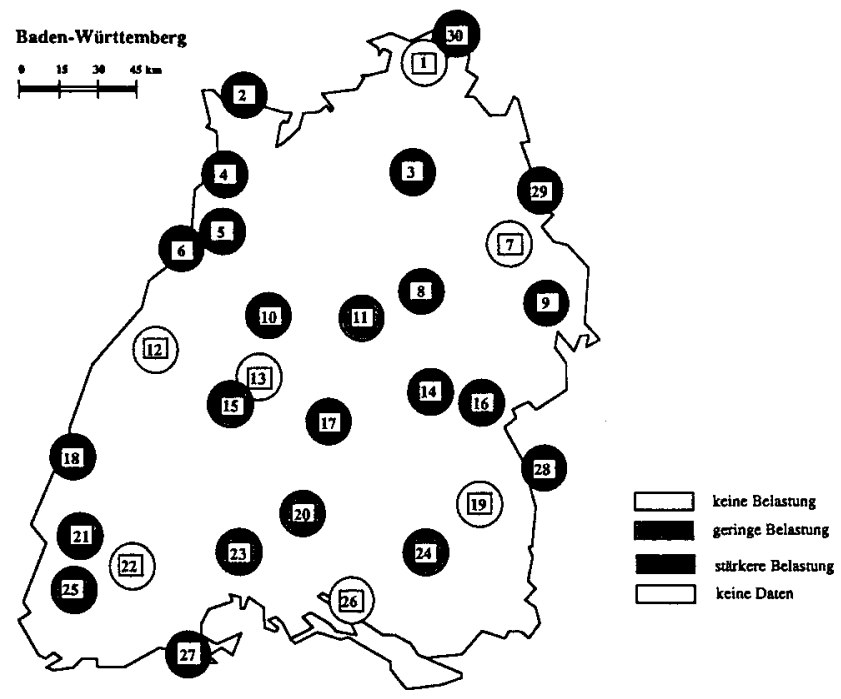

Abb. 2: Darstellung der Ergebnisse des Klon-Fichten-Meßprogramms 1989 für Baden-Württemberg

Flechtentafeln und -kartierungen erfolgte eine Beprobung von Standortfichten. Die Nadelproben wurden auf die organischen Schadstoffkomponenten PAH und PCB analysiert. Eine vergleichbar umfassende Untersuchung liegt bislang noch für keine andere Region Baden-Württembergs vor. Derzeit werden die Meßergebnisse ausgewertet.

Die im Verlauf des Jahres 1989 erhobenen und ausgewerteten Daten der verschiedenen Meßnetze führen zu einer deutlichen Aufteilung Baden-Württembergs in zwei große regionale Belastungszonen. Die Ergebnisse lassen einen stärkeren Immissionseinfluß für den nördlichen gegenüber dem südlichen Teil des Landes erkennen.

\section{Fortführung und Weiterentwicklung}

Im Zusammenhang mit der Neuorganisation der Landesanstalt für Umweltschutz sollen die bisherigen inmissionsökologischen Erhebungen auf ökotoxikologische Untersuchungen ausgebaut werden, insbesondere bezüglich der organischen Schadstoffe.

Weiterhin gefördert werden soll die Zusummenarbeit mit anderen Bundesländern. Ein Beispiel ist das länderübergreifende KlonFichten-Programm von Baden-Württemberg und Bayern. Auf der Basis gleicher Verfahrensrichtlinien besteht seit 1986 ein Bioindikatormeßnetz für den gesamten süddeutschen Raum. Im Sinne einer Vergleichbarkeit von Wirkungserhebungen müssen bundeseinheitliche Methodenvorschriften zur Bioindikation erarbeitet werden.

\section{Literatur}

Debus, R.; Dittrich, B.; Schröder, P. \& Vollmer J.: Biomonitoring organischer Schadstoffe. Handbuch des Umweltschutzes, 45. Erg. Lfg., Kap. II -1.7 , ecomed verlagsgesellschaft mbh, Landsberg - München · Zürich 1989

LFU-Landesanstalt für Umweltschutz (Hrsg.): Immissionsökologisches Wirkungskataster Baden-Würtemberg. Jahresbericht 1988 der Landesanstalt für Umweltschutz (Institut für Ökologie und Naturschutz), 237 S., Karlsruhe 1989

SCHINNER, W.: Gift in der Pfanne. Pirsch 4, $228-229$ (1984)

ZIMMERMANN, R.-D.: Immissionsökologisches Wirkungskataster Baden-Württemberg. UWSF-Z. Umweltchem. Ökotox. 1 (4) $57-58$ (1989)

Dr. R.-D. Zimmermann, LFU Karlsruhe 